\title{
The prophylactic effect of bovine colostrum on respiratory infection and diarrhea in formula-fed infants: a randomized trial
}

\section{Ke Chen (D263662086@qq.com)}

Department of Nutrition, Chengdu women's \& Children's central hospital, Chengdu https://orcid.org/0000-0001-7455-0900

\section{Haixia Chen}

Section of Disease Prevention and Control, Baoxing Central for Disease Control and Prevention

Jinwen Luo

Department of Child Health Care, Liucheng Community Healthcare Center

Chenggui Zeng

Department of Child Health Care, Caojiaxiang Community Healthcare Center

\section{Xiaobing Dong}

Department of Child Health Care, Hehuachi Community Healthcare Center

\section{Min Zhou}

Department of Child Health Care, Jitouqiao Community Healthcare Center

Changqi Liu

School of Exercise and Nutritional Science, San Diego State University

\section{Research}

Keywords: ovine colostrum, infant, morbidity, prophylaxis

Posted Date: January 7th, 2020

DOI: https://doi.org/10.21203/rs.2.20117/v1

License: (1) (i) This work is licensed under a Creative Commons Attribution 4.0 International License. Read Full License 


\section{Abstract}

\section{Background}

It has been reported that regular consumption of bovine colostrum has a protective role for the gastrointestinal immunity of infants. However, not enough investigation has explored the prophylactic effect of bovine colostrum on the morbidity of diarrhea and respiratory tract infections in formula-fed infants. The purpose of the study is to explore the prophylactic effect of BC on morbidity of diarrhea and respiratory tract infections in formula-fed infants.

\section{Methods}

A total of 192 term infants aged 6 to 9 months, who were previously fully formula-fed after birth, were randomly recruited into this multi-centric, randomized, blank-controlled, opened, and post-marketing intervention study. Infants in the intervention group (IG) were given a commercially available bovine colostrum sachet once a day for 3 months while infants in the control group (CG) were not given the bovine colostrum sachet. The morbidity and duration of diarrhea and respiratory tract infections and the fecal IgA, calprotectin, and total fatty acid were monitored during the intervention.

\section{Result}

There was a lower incidence of loose stool [IG/CG rate ratio: $0.22,95 \% \mathrm{Cl}: 0.09-0.67]$, increased stool frequency $(0.30,0.17-0.78)$, loss of appetite $(0.28,0.11-0.47)$, sneezing $(0.22,0.07-0.69)$, upper respiratory infection $(0.55,0.32-0.88)$, and diarrhea $(0.25,0.09-0.61)$ and shorter duration of diarrhea for infants in IG than those in CG.

\section{Conclusion}

Bovine colostrum intervention for 3 months showed a potential prophylactic effect on respiratory infection and diarrhea in formula-fed infants. The present data may be applicable to other infants of similar socioeconomic status.

\section{Background}

The Child Health Epidemiology Reference Group of World Health Organization (WHO) and United Nations International Children's Emergency Fund (UNICEF) estimated that of 6.3 million deaths in children younger than 5 years in $2013,51.8 \%$ (3.257 million) were attributable to infectious causes ${ }^{[1]}$. Respiratory diseases are a major cause of morbidity and mortality in developing countries. Diarrhea, on the other hand, is a major killer disease in children under five, and recurrent diarrhea affects nearly $20 \%$ of the population ${ }^{[2]}$. In China, from 2003 to 2012, a total of 127,539 deaths from diarrhea were reported among children $<5$ years of age, of whom an estimated $42 \%(53,559)$ had an illness caused by rotavirus ${ }^{[3]}$. 
Defense against infectious disease is a matter of the highest importance for the health and development of children in developing countries. Breastfeeding is the most cost-effective intervention for protecting children against infectious diseases and all causes of mortality ${ }^{[4]}$. It is generally assumed that the protection is due to multiple anti-infective, anti-inflammatory, and immune regulatory factors transmitted through milk ${ }^{[5,6]}$. However, oral intake of exogenous immunoglobulins is not effective in preventing infectious disease ${ }^{[7]}$. A possible reason is that anti-infective factors act in synergy, and a single agent may not be effective.

Commercially available bovine colostrum (BC) has high concentrations of anti-infective factors such as immunoglobulins, lactoferrin, lactoperoxidase, insulin-like growth factors, and transforming growth factors. These components have substantial homology to their human counterparts ${ }^{[8,9]}$. It has been reported that regular consumption of $\mathrm{BC}$ has a protective role for the gastrointestinal immunity of infants due to its direct effect on the stimulation and development of gut-associated lymphoid tissues, which are mainly responsible for enhancing the infantile gut immunity ${ }^{[10]}$. The studies in infants suggested the possible therapeutic and prophylactic role of orally ingested $\mathrm{BC}$ in control of many bacterial and viral gastrointestinal infectious diseases ${ }^{[11]}$.

However, not enough investigation has explored the prophylactic effect of BC on the morbidity of diarrhea and respiratory tract infections (RTIs) in formula-fed infants. We carried out a randomized, blankcontrolled, and opened trial in multiple suburban communities of Sichuan Province to evaluate the role of $B C$ in the prevention of diarrhea and RTIs. In this study, we hypothesized that formula-fed infants supplemented with $\mathrm{BC}$ would have reduced morbidity of diarrhea and RTIs compared with infants who only receive formulas without $\mathrm{BC}$.

\section{Methods}

Participants and ethical approval

This multi-centric, randomized, blank-controlled, opened, and post-marketing intervention study was performed in the Baoxing county of Ya'an city and the Wenjiang, Jinniu, and Wuhou districts of Chengdu City, Sichuan Province in western China from August 2017 to February 2018. A total of 192 term infants aged 6 to 9 mo, who were previously fully formula-fed after birth, were randomly recruited from five health care facilities in the four described regions.

We conducted a preliminary survey in each health care facility to identify families with a fully formula-fed infant aged 6 to $9 \mathrm{mo}$. Then, field health workers conducted a food-intake survey to confirm that the infants were truly fully fed on formula. Eligible families were visited by a fieldworker who explained the protocol, answered questions, and obtained written informed consent from the parents. The enrollment and research plan were reviewed and approved by the institutional ethics committee of the Angel Children's Hospital Chengdu (Sichuan, China). The present study complied with the code of ethics of the World Medical Association (Declaration of Helsinki). 
Eligibility and exclusion criteria

The eligibility criteria for participation were as follows:

1. Apparent good health without common obstetric risk factors;

2. Full-term with 37-42 weeks of gestation at birth and birth weight of more than $2500 \mathrm{~g}$;

3. Previously fully formula-fed after birth and aged 6 to 9 mo at recruitment;

4. Parent or guardian approval for participating in all aspects of the study;

5. Parent or guardian agreement to avoid additional BC, probiotics, and bovine lactoferrin products during the investigation;

6. Infants who are receiving regular health care in local health care facilities.

Exclusion criteria included a history of severe, persistent, or chronic diarrhea; severe malnutrition; serious infections requiring hospitalization in the prior month; serious chronic illness; or a personal or family history of allergy to cow's milk or intact infant formula, eczema, allergic rhinitis, or asthma; and diagnosed lactose intolerance at recruitment.

Participation in the study was voluntary, and the parents have the right to withdraw their child from the study without providing a reason and with no loss of benefits to which the child is entitled. If a parent chose to withdraw their child, the study personnel must be informed immediately. The investigator has the right to terminate the participation of any child at any time if they deem it to be in the best interest of the child. Examples of possible reasons for premature withdrawal of a study subject include: child's parents withdrew consent for personal reasons, child's general condition contraindicated continuing the study (such as allergy to BC and lactose intolerance) as judged by the study personnel or the medical expert, significant non-compliance with study protocol or lack of cooperation, Serious Adverse Event (SAE), and lost to follow-up.

Primary and secondary outcomes

The primary outcome of the present study was the morbidity of diarrhea and RTIs in formula-fed infants during the intervention. The secondary outcome was the effect of the intervention on the duration of respiratory- and diarrhea-related illnesses, growth of the infants, family functioning scores, and the levels of fecal IgA, calprotectin, and total fatty acids.

Sample size

According to similar studies ${ }^{[12]}$, a $5 \%$ absolute increase in the longitudinal prevalence of diarrhea is associated with an increased risk for mortality of $17 \%$. Thus, we considered a $5 \%$ difference in the longitudinal prevalence of diarrhea among groups to be clinically meaningful. We estimated that 80 infants per group would ensure a power of $95 \%(a=0.05)$ to detect between-group differences for the longitudinal prevalence of diarrhea (log-transformed). To account for potential participant attrition, we recruited 96 subjects for each group. That is to say, the initial sample size (192 in total) allowed sufficient 
power (>95\%) to evaluate the incidence of infectious illnesses as the primary outcome in the present study.

Intervention

Eligible infants were randomly assigned to one of the two groups. Infants in the intervention group (IG) were given a commercially available BC sachet (Hainan Govking High-Tech Dairy Industry Co., Ltd, Haikou, China,Product Batch Number: D7010) once a day, which contained a bovine IgG concentration of $200 \mathrm{mg} / \mathrm{g}$ (Table 1). Each sachet $(1.0 \mathrm{~g})$ can be mixed with $30-50 \mathrm{~mL}$ of warm water (below $40^{\circ} \mathrm{C}$ ), or added to milk, rice cereal, soup, rice porridge, or other foods before ingestion. Infants in the control group (CG) were not given the $\mathrm{BC}$ sachet. The intervention duration was three months.

Table 1

Nutritional ingredients of pure bovine

colostrum milk powder ( $1 \mathrm{~g} /$ sachet).

\begin{tabular}{|lll|}
\hline Items & Per 100 g & RNV\% \\
\hline Energy (kJ) & 1534 & $18 \%$ \\
\hline Protein (g) & 58.8 & $98 \%$ \\
\hline Fat (g) & 1.3 & $2 \%$ \\
\hline Carbohydrate (g) & 28.6 & $10 \%$ \\
\hline Sodium (mg) & 400 & $20 \%$ \\
\hline IgG (g) & 20.4 & \\
\hline Lactoferrin (mg) & 171 & \\
\hline RNV, relative nutritive value. \\
\hline
\end{tabular}

Randomization and allocation concealment

Immediately after recruitment, each infant was assigned a study number that had been previously, completely randomly assigned to the IG or CG with fixed, equal allocation to each group prepared by a research secretary at the Chengdu Women's and Children's Central Hospital, who was not connected to the study. The RAND function of Excel (Microsoft, Redmond, WA, USA) was used to generate randomly permutated codes with concealment to ensure that the allocation was not made before the parents of the infants had given their consent and joined the study. The physicians responsible for enrollment of patients allocated the next available number on entry into the trial. At enrollment, the parent of the patient received a closed envelope containing a written usage for this trial.

Blinding 
The laboratory personnel, data manager and statistician were blinded to the treatment assignment of each infant throughout the study period until the end of the data analysis.

Anthropometric measurements

Anthropometric examinations for each infant were conducted by the same trained nurse at baseline and follow-up (3 months) using standardized techniques to eliminate the inter-examiner error. Duplicate measurements were performed for all infants. The inter-examiner coefficient of variation of weight, length, and head circumference $(\mathrm{HC})$ measurement for each examiner in IG and $C G$ was $<5 \%$. Weight was recorded using a weighing scale (100 Med, Beijing, China) to the nearest $100 \mathrm{~g}$ with infants in minimal clothing and bare feet. Similarly, length was measured in the standard supine position by a supine scale (Haode, Guangzhou, China) to the nearest $0.1 \mathrm{~cm}$.

Fecal sample collection and biochemical assessment

Fecal samples were randomly collected from a subset of 60 infants (30 from IG and 30 from CG) before and after the intervention for measurement of fecal IgA, calprotectin, and total fatty acids using commercial enzyme-linked immunosorbent assays (ELISA; Sunbiote, Shanghai, China).

Household and nursery surveillance for morbidity

Baseline morbidity data were based on self-reported infection status via questionnaire, obtained through interviews with parents or caregivers of the infants, in the previous 2 weeks. The questionnaire included questions on demographics, family socioeconomic status related to the educational levels of the main caregivers, family monthly income, and use of vitamin/mineral supplement before the intervention. Parents or guardians were requested to inform the health care workers if their infant had experienced any of the symptoms on the standardized checklist. The symptoms recorded included respiratory-related illnesses (at least including one of the symptoms: rhinorrhea, cough, wheezing, or nasal congestion), diarrhea-related illnesses (at least including one of the symptoms: diarrhea, vomiting, or nausea), and other illnesses. Diarrhea was defined as the passage of loose or liquid stools with a high stool frequency (i.e., > 3 stools daily). A morbidity episode was defined as an event of morbidity symptoms with three illness-free days between events. Before and after the intervention, the parents or caregivers completed the PedsQL ${ }^{\mathrm{T}}$ 2.0 Family Impact Module. All adverse events, defined as any untoward medical occurrence in an infant during the intervention period, were recorded.

\section{Compliance}

Compliance with the $\mathrm{BC}$ supplementation was monitored using a recording table, in which parents or caregivers recorded the number of sachets taken for the entire day. Distribution (performed by fieldworkers) and consumption of the sachet were under close supervision. Information on the acceptability of the $\mathrm{BC}$ was obtained by a short questionnaire after administration. After the intervention, the fieldworker received the short questionnaire to examine and review the quantity of BC consumed. 
Data were presented as mean \pm standard deviation (SD) for normally distributed variables or median (25th, 75th percentiles) for abnormally distributed variables; when necessary, data were normalized using natural log-transformations. Tests of significance were two-tailed and $\mathrm{P}<0.05$ was considered statistically significant. Student's t-test was used to compare the data with normal distribution and homogeneous variance before and after the intervention between the two groups, and the Wilcoxon signrank test was used for abnormally distributed data. The $\chi 2$ test was used for categorical variables between the groups. Negative binomial regression was used to estimate the effectiveness of the intervention for morbidity episodes. For each child, an average duration of illness was calculated as the total number of days with the illness divided by the number of episodes of the illness. Data were analyzed using SAS 9.0 for Windows (SAS Institutes, Cary, NC, USA).

\section{Results}

About $8.85 \%$ (17 of 192) of the infants dropped out during the course of the study. Eleven of these infants were lost to follow up, four for data error and one for withdrawal due to rotavirus enteritis. Thus, the morbidity of the infectious diseases and the anthropometric indices were obtained from 175 infants (86 for IG and 89 for $C G$ ) at baseline and after the intervention (Fig. 1).

\section{Baseline characteristics}

There were no significant differences in sex composition, birth weight, age, weight, length, and $\mathrm{HC}$ at recruitment, years of education of main caregivers, monthly average household income, living environment, numbers of household members, and complementary feeding between the two groups (all $P$ values $>0.05$; Table 2 ). There was no any important harms or unintended effects in each group during the intervention. 
Table 2

Comparison of the clinical and socioeconomic data of the infants (mean $\pm S D$ ).

\begin{tabular}{|c|c|c|c|c|}
\hline Items & $\begin{array}{l}\text { IG }(n= \\
86)\end{array}$ & $\begin{array}{l}C G(n= \\
89)\end{array}$ & $\begin{array}{l}t / \chi^{2} \\
\text { value }\end{array}$ & $\begin{array}{l}\mathrm{P} \\
\text { value }\end{array}$ \\
\hline Female[n(\%)]* & $38(44.2)$ & $42(47.2)$ & 0.159 & 0.6899 \\
\hline Age (months)* & $\begin{array}{l}10.18 \pm \\
2.6\end{array}$ & $\begin{array}{l}10.14 \pm \\
3.2\end{array}$ & 0.091 & 0.928 \\
\hline Birth weight $(\mathrm{kg})^{*}$ & $3.31 \pm 0.4$ & $3.27 \pm 0.5$ & 0.583 & 0.7197 \\
\hline Length when recruited $(\mathrm{cm})^{\star}$ & $\begin{array}{l}74.19 \pm \\
5.4\end{array}$ & $\begin{array}{l}73.34 \pm \\
5.2\end{array}$ & 1.061 & 0.2902 \\
\hline Weight when recruited $(\mathrm{kg})^{*}$ & $9.20 \pm 1.2$ & $9.23 \pm 1.9$ & -0.124 & 0.9012 \\
\hline $\mathrm{HC}$ when recruited $(\mathrm{cm})^{\star}$ & $\begin{array}{l}45.47 \pm \\
1.9\end{array}$ & $\begin{array}{l}44.96 \pm \\
2.0\end{array}$ & 1.728 & 0.0858 \\
\hline Levels of education of main caregivers $(n)^{\star}$ & & & 3.142 & 0.3702 \\
\hline Junior middle school or below & 25 & 24 & & \\
\hline High school & 12 & 13 & & \\
\hline Junior college & 17 & 27 & & \\
\hline University or above & 32 & 25 & & \\
\hline $\begin{array}{l}\text { Levels of monthly household income per capita } \\
\text { (yuan)* }\end{array}$ & & & 6.459 & 0.2641 \\
\hline$<500$ & 1 & 0 & & \\
\hline $501-1000$ & 24 & 19 & & \\
\hline $1001-1500$ & 9 & 7 & & \\
\hline $1501-2000$ & 3 & 11 & & \\
\hline $2001-3000$ & 8 & 8 & & \\
\hline$>3000$ & 41 & 44 & & \\
\hline Living environment* & & & 0.002 & 1 \\
\hline Rural & 48 & 50 & & \\
\hline Urban & 38 & 39 & & \\
\hline Numbers of household members $(n)^{\star}$ & & & 2.193 & 0.1386 \\
\hline
\end{tabular}

IG, bovine colostrum intervention group; CG, blank control group; *, no significant differences between the two groups $(P>0.05)$. 


\begin{tabular}{|c|c|c|c|c|}
\hline Items & $\begin{array}{l}\text { IG }(n= \\
86)\end{array}$ & $\begin{array}{l}C G(n= \\
89)\end{array}$ & $\begin{array}{l}t / \chi^{2} \\
\text { value }\end{array}$ & $\begin{array}{l}P \\
\text { value }\end{array}$ \\
\hline$<4$ & 16 & 25 & & \\
\hline$\geq 4$ & 70 & 64 & & \\
\hline Complementary feeding when recruited $(n)$ * & & & 0.001 & 1 \\
\hline Yes & 85 & 88 & & \\
\hline No & 1 & 1 & & \\
\hline
\end{tabular}

Primary outcome: Effect on morbidity

Morbidity data were collected for a median of $90 \mathrm{~d}$ for the two groups. Table 3 presents the incidence of morbidity episodes at follow-up. 
Table 3

Incidence of morbidity episodes at follow-up

\begin{tabular}{|c|c|c|c|c|}
\hline \multirow[t]{2}{*}{ Items } & \multicolumn{2}{|l|}{ IG $(n=86)$} & \multicolumn{2}{|l|}{ CG $(n=89)$} \\
\hline & $\begin{array}{l}\text { Morbidity } \\
\text { events }\end{array}$ & $\begin{array}{l}\text { Incidence per } 100 \\
\text { child dayst }\end{array}$ & $\begin{array}{l}\text { Morbidity } \\
\text { events }\end{array}$ & $\begin{array}{l}\text { Incidence per } 100 \\
\text { child days } ¥\end{array}$ \\
\hline Cough & 39 & 0.50 & 40 & 0.50 \\
\hline Runny nose & 43 & 0.56 & 48 & 0.60 \\
\hline Nasal congestion & 29 & 0.37 & 28 & 0.35 \\
\hline Fever $\left(\geq 37.5^{\circ} \mathrm{C}\right)$ & 8 & 0.10 & 8 & 0.10 \\
\hline Loose stool & 5 & 0.06 & 14 & 0.17 \\
\hline $\begin{array}{l}\text { Increased stool } \\
\text { frequency }\end{array}$ & 3 & 0.04 & 6 & 0.07 \\
\hline Choking on milk & 0 & 0 & 0 & 0 \\
\hline Loss of appetite & 0 & 0 & 3 & 0.04 \\
\hline Wheezing & 0 & 0 & 0 & 0 \\
\hline Sneezing & 1 & 0.01 & 2 & 0.02 \\
\hline $\begin{array}{l}\text { Upper respiratory } \\
\text { infection* }\end{array}$ & 10 & 0.13 & 9 & 0.11 \\
\hline Pneumonia* & 0 & 0 & 0 & 0 \\
\hline Diarrhea* & 8 & 0.10 & 20 & 0.25 \\
\hline
\end{tabular}

IG/CG rate ratios for respiratory- and diarrhea-related illnesses and their symptoms are presented in Table 4. There was a lower incidence of loose stool, increased stool frequency, loss of appetite, sneezing, upper respiratory infection, and diarrhea for infants in IG than infants in CG $(P<0.05)$. 
Table 4

IG/CG rate ratios for respiratory- and diarrhea-related illnesses and their symptoms with estimates of the intervention effect.

\begin{tabular}{|lll|}
\hline Item & Rate ratio (IG/CG) & $95 \% \mathrm{Cl}$ \\
\hline Cough & 0.55 & $0.24,1.77$ \\
\hline Runny nose & 0.43 & $0.19,2.01$ \\
\hline Nasal congestion & 0.61 & $0.31,1.56$ \\
\hline Fever $\left(\geq 37.5^{\circ} \mathrm{C}\right)$ & 0.78 & $0.29,2.55$ \\
\hline Loose stoolt & 0.22 & $0.09,0.67$ \\
\hline Increased stool frequency $\dagger$ & 0.30 & $0.17,0.78$ \\
\hline Choking on milk & - & - \\
\hline Loss of appetitet & 0.28 & $0.11,0.47$ \\
\hline Wheezing & - & - \\
\hline Sneezing $\dagger$ & 0.22 & $0.07,0.69$ \\
\hline Upper respiratory infection* & 0.55 & $0.32,0.88$ \\
\hline Pneumonia* & - & - \\
\hline Diarrhea* & 0.25 & $0.09,0.61$ \\
\hline $\begin{array}{l}\text { IG, bovine colostrum intervention group; CG, blank control group; }{ }^{*} \text {, diagnosed by local doctors; } \\
\text { significant differences between the two groups. }\end{array}$ & \\
\hline
\end{tabular}

Secondary outcome: Effect on the morbidity duration

The duration of diarrhea in IG was significantly shorter than that in $C G(P<0.05)$ (Table 5). However, the intervention did not show a noticeable effect on the duration of other morbidities $(P>0.05)$. 
Table 5

Estimates of intervention effect for average duration of morbidity in days [day, median (p25, p75)].

\begin{tabular}{|lllll|}
\hline Items & IG $(\mathbf{n}=\mathbf{8 6})$ & $\mathbf{C G}(\mathbf{n}=\mathbf{8 9})$ & Z value & P value \\
\hline Cough & $0(0,3)$ & $0(0,4)$ & 0.0945 & 0.9247 \\
\hline Runny nose & $0(0,4.5)$ & $0(0.4)$ & 0.0502 & 0.9600 \\
\hline Nasal congestion & $0(0,0)$ & $0(0,0)$ & 0.1689 & 0.8659 \\
\hline Fever $\left(\geq 37.5^{\circ} \mathrm{C}\right)$ & $0(0,0)$ & $0(0,0)$ & 0.1364 & 0.8915 \\
\hline Loose stool & $0(0,0)$ & $0(0,0)$ & 1.4627 & 0.1435 \\
\hline Increased stool frequency & $0(0,0)$ & $0(0,0)$ & -0.7811 & 0.4347 \\
\hline Choking on milk & $0(0,0)$ & $0(0,0)$ & 0.0000 & 1.0000 \\
\hline Loss of appetite & $0(0,0)$ & $0(0,0)$ & -1.7058 & 0.0881 \\
\hline Wheezing & $0(0,0)$ & $0(0,0)$ & 0.0000 & 1.0000 \\
\hline Sneezing & $0(0,0)$ & $0(0,0)$ & -0.5310 & 0.5954 \\
\hline Upper respiratory infection* & $0(0,0)$ & $0(0,0)$ & 0.3688 & 0.7122 \\
\hline Pneumonia* & $0(0,0)$ & $0(0,0)$ & 0.0000 & 1.0000 \\
\hline Diarrhea* & $0(0,0)$ & $0(0,3)$ & -2.2162 & 0.0267 \\
\hline IG, bovine colostrum intervention group; CG, blank control group; ${ }^{*}$, diagnosed by locative doctors; & & \\
\hline significant differences between the two comparison groups. & & & \\
\hline
\end{tabular}

Secondary outcome: Effect on the anthropometric indices

The present study showed that there was no significant effect of the $B C$ intervention on the infant length, weight, and $\mathrm{HC}$ measurements $(P>0.05)$ (Table 6). 
Table 6

Effect of the intervention on the infant anthropometric indices (Mean \pm SD).

\begin{tabular}{|c|c|c|c|c|}
\hline Items & IG $(n=86)$ & $C G(n=89)$ & t values & $P$ values \\
\hline \multicolumn{5}{|c|}{ Length $(\mathrm{cm})$} \\
\hline $\mathrm{BIt}$ & $74.19 \pm 5.4$ & $73.34 \pm 5.2$ & 1.061 & 0.2902 \\
\hline Alt & $77.52 \pm 5.3$ & $77.00 \pm 5.0$ & 0.668 & 0.5052 \\
\hline \multicolumn{5}{|c|}{ Weight (kg) } \\
\hline $\mathrm{BIt}$ & $9.20 \pm 1.2$ & $9.23 \pm 1.9$ & -0.124 & 0.9012 \\
\hline Alt & $10.90 \pm 1.3$ & $10.89 \pm 2.0$ & 0.039 & 0.9688 \\
\hline \multicolumn{5}{|c|}{$\mathrm{HC}(\mathrm{cm})$} \\
\hline $\mathrm{Blt}$ & $45.47 \pm 1.9$ & $44.96 \pm 2.0$ & 1.728 & 0.0858 \\
\hline Alt & $46.63 \pm 1.8$ & $46.22 \pm 1.8$ & 1.506 & 0.1338 \\
\hline
\end{tabular}

Secondary outcome: Effect on the fecal biochemical indices

After BC intervention, the levels of fecal calprotectin of infants in IG was significantly lower than that of infants in $C G(P<0.5)$, but there was no significant effect on the fecal fatty acids and slgA levels $(P>$ 0.05) (Table 7). 
Table 7

Effect of intervention on the infant fecal biochemical indices (Mean \pm SD).

\begin{tabular}{|c|c|c|c|c|}
\hline Items & IG $(n=30)$ & $C G(n=30)$ & t values & $P$ values \\
\hline \multicolumn{5}{|c|}{ Calprotectin $(\mu \mathrm{g} / \mathrm{mL})$} \\
\hline Blt & $545.28 \pm 59.0$ & $556.96 \pm 54.9$ & 0.794 & 0.431 \\
\hline Alł & $411.66 \pm 81.1$ & $373.34 \pm 58.6$ & 2.098 & 0.040 \\
\hline \multicolumn{5}{|c|}{ Fatty acid $(\mu \mathrm{mol} / \mathrm{L})$} \\
\hline Blt & $3.28 \pm 0.4$ & $3.43 \pm 0.34$ & 1.565 & 0.123 \\
\hline Alt & $4.02 \pm 0.4$ & $4.20 \pm 0.5$ & 1.54 & 0.129 \\
\hline \multicolumn{5}{|c|}{$\lg A(\mu \mathrm{g} / \mathrm{mL})$} \\
\hline Blt & $125.27 \pm 12.1$ & $120.12 \pm 9.0$ & 1.871 & 0.066 \\
\hline Alt & $147.77 \pm 13.8$ & $152.19 \pm 13.5$ & 1.254 & 0.215 \\
\hline
\end{tabular}

Secondary outcome: Effect on the PedsQLTM Family Impact Subscale

The present study did not find a significant difference in the PedsQLTM Family Impact Subscale between the two groups before the intervention $(P>0.05)$ (Table 8$)$. The emotional and social functioning scores of infants in IG were markedly improved after the BC intervention when compared with the infants in CG (all $P<0.05$ ). However, no significant difference of the physical functioning score was found between the two groups after the $B C$ intervention $(P>0.05)$. 
Table 8

Effect of intervention on the PedsQLTM Family Impact Subscale (Mean \pm SD).

\begin{tabular}{|c|c|c|c|c|}
\hline Items & IG $(n=86)$ & $C G(n=89)$ & t values & $P$ values \\
\hline \multicolumn{5}{|c|}{ Physical functioning } \\
\hline Blt & $11.41 \pm 5.1$ & $11.06 \pm 5.1$ & 0.454 & 0.6506 \\
\hline Alt & $8.70 \pm 3.3$ & $9.13 \pm 3.7$ & -0.81 & 0.4188 \\
\hline \multicolumn{5}{|c|}{ Emotional functioning } \\
\hline $\mathrm{Blt}$ & $8.58 \pm 4.1$ & $8.12 \pm 3.7$ & 0.78 & 0.4366 \\
\hline Al‡ & $6.12 \pm 2.0$ & $7.01 \pm 3.0$ & -2.301 & 0.0226 \\
\hline \multicolumn{5}{|c|}{ Social functioning } \\
\hline Blt & $6.55 \pm 3.0$ & $6.35 \pm 2.9$ & 0.448 & 0.6544 \\
\hline Al‡ & $4.63 \pm 1.7$ & $5.30 \pm 2.2$ & -2.249 & 0.0258 \\
\hline
\end{tabular}

\section{Discussion}

Several potential benefits of BC supplementation were observed in formula-fed infants. There was a significantly lower incidence of loose stool, increased stool frequency, loss of appetite, sneezing, upper respiratory infection, and diarrhea and shorter duration of diarrhea for infants receiving 3-month BC supplementation. Some studies have reported adverse reactions to BC such as nausea, vomiting, headache, insomnia, pruritus, and myalgia, which might be caused by the allergy to the protein components in $\mathrm{BC}^{[13-15]}$. The present investigation and many other studies ${ }^{[16-18]}$ did not find any adverse reaction or intolerance to $\mathrm{BC}$. Thus, $\mathrm{BC}$ is safe to be given to infants and children without allergy or intolerance to cow's milk.

\section{Prophylactic effect on morbidity}

The use of whole $\mathrm{BC}$ in addition to standard treatment for the recurrent or acute respiratory infection and diarrhea has been reported in previous studies in many countries. BC supplementation has consistently and significantly reduced the symptoms of respiratory infection and diarrhea, shortened the morbidity duration, and accelerated the recovery time ${ }^{[19-23]}$. However, to our knowledge, there has been limited investigation of the prophylactic effect of $\mathrm{BC}$ on morbidity of respiratory infection or diarrhea in formulafed infants. 
Some studies have reported $100 \%$ protection of infants by BC against symptomatic rotavirus infection, including the four known human rotavirus serotypes ${ }^{[24,25]}$. Ebina ${ }^{[26]}$ reported that infants who received Rota colostrum for two weeks did not develop rotavirus-induced diarrhea, indicating that oral administration of $B C$ is an effective and safe mean of preventing diarrhea. Another study reported the use of oral bovine immunoglobulin concentrate with high antibody titer against Escherichia coli as an effective method for the prevention of enteropathogenic E. coli-induced diarrhea ${ }^{[23]}$. Bogstedt et a1. ${ }^{[27]}$ demonstrated sufficient prophylactic activity of a daily dose of $500 \mathrm{mg}$ bovine immunoglobulins against rotavirus-induced diarrhea. Similarly, 200-400 mg lozenges of bovine colostrum were found to be effective in improving and modulating the humoral and cellular immune indices during the feeding (2 weeks) and post-feeding (2 weeks) periods ${ }^{[28]}$.

Knowledge of the effects of $\mathrm{BC}$ for prevention of infections in children is limited. In the present study, the incidence of the respiratory and gastrointestinal tract infections was significantly lower after 3 months of $\mathrm{BC}$ supplementation in formula-fed infants. Our results were in agreement with previous reports.

The dosage of oral $\mathrm{BC}$ administration needed to obtain a therapeutic effect awaits to be determined, and the ideal duration of $\mathrm{BC}$ treatment in an "at risk" population remains unknown. Previous studies have investigated daily oral intake of $200 \mathrm{mg}$ to $40 \mathrm{~g}$ of dried BC for 10 days to 3 months ${ }^{[11]}$. The present study demonstrated that daily intake of $200 \mathrm{mg}$ of immunoglobulins for 3 months was beneficial for the prophylaxis against respiratory infection and diarrhea.

Effect on the anthropometric indices

To our knowledge, very few studies have explored the effect of BC on the linear growth of children. Ylitalo et al. showed that ${ }^{[29]}$ infants and children suffered from rotavirus-caused acute diarrhea had a significant increase in body weight $(403 \mathrm{~g}$ ) after given bovine colostrum for 4 days as compared to the control group (343 g). However, the study performed by Suwarba ${ }^{[18]}$ showed no effect of BC intervention on the children's body weight, which was in agreement with our study.

It is important to elucidate the role of $\mathrm{BC}$ intervention in physical growth and development of infants. We could not explain why the BC intervention exhibited different effects on the linear growth of children. Whether the discrepancy was caused by an insufficient dosage or intervention duration that failed to compensate for the deleterious effect of the diseases, the age of infants, or other non-homogeneous factors remains unclear. Therefore, future studies are warranted to compare different fortification methods for the anthropometric status of formula-fed infants.

Effect on the fecal biochemical indices

Calprotectin is abundant in the cytosolic fraction of neutrophils and has important extracellular activities $^{[30]}$. It has been shown to have bactericidal and fungicidal properties and to play a role in the regulation of the inflammatory process ${ }^{[31,32]}$. Studies have reported that the mean fecal calprotectin was higher in exclusively breastfed infants in the first and sixth months than formula- and mix-fed infants, 
and the protective role of breastfeeding may be attributed to immune mediators such as calprotectin [3335]. Based on the role of calprotectin in inflammation, its abundance in exclusively breastfed infants may be a sign of an enhanced mucosal immune maturity. The higher levels of fecal calprotectin in infants after $\mathrm{BC}$ intervention as compared to infants who did not receive $\mathrm{BC}$ explained the improved gastrointestinal health.

Considering the critical role of $\lg A$ in mucosal immunity $[35,36]$, we hypothesized that $B C$ supplementation might have an impact on the development of the immune system in infants. However, we did not observe a significant change in the fecal slgA levels as a result of the $B C$ intervention in the present study. Besides the heterogeneity of the racial composition and the laboratory methodology and the bias effect of the uncontrolled factors mentioned above, it is also possible that $B C$ has limited influence on the metabolic pathway of slgA. Thus, more investigations are needed to explore the correlation between fecal inflammatory and immunological markers and BC supplementation.

Furthermore, the amount and composition of fecal fatty acids reflect fat ingestion, intestinal fatty acid absorption, and the activity of colonic bacteria ${ }^{[37,38]}$. The lack of significant difference of the fecal slgA and fatty acid in infants between the two groups might indicate that the improvement of the intestinal symptoms may not correlate with the slgA secretion and fatty acid absorption.

Strength and limitation analysis of the present study

We believe that the present open-blinded, randomized, and controlled trial had many strengths. The two groups were comparable at baseline for all objective indices. Infants were randomly assigned to one of the groups by an investigator who was external to recruitment and had no information regarding potential covariate factors. Because of large sample size and low attrition, the results can be generalized to children in western China of similar socioeconomic conditions and dietary patterns.

Despite these advantages, the present study was not without limitations. Because the $B C$ was provided for free, the subjective tendencies of the parents and guardians might affect the objectivity of the questionnaires and may have introduced bias. However, most of the study factors were objective indicators, which reduced the bias as much as possible. Furthermore, because of the limited design of the original protocol, we did not evaluate the effect of other micronutrients ${ }^{[39,40]}$ (such as zinc, iron, vitamin A, and probiotics) on morbidity. Measuring and evaluating these micronutrient levels will be an important addition in future studies. Moreover, the duration of fortification in the present study is only three months. Long-term effects of $\mathrm{BC}$ on infant health may be observed with an extension of the fortification time. Finally, although it has taken us about 7 months to complete all the field interventions, the beginning and ending dates between the two groups in four sub-centers were almost the same, which might minimize the seasonal and climatic bias. In future study design, the seasonal and climatic bias will be taken into consideration.

\section{Conclusion}


In summary, our data indicated that the BC intervention for 3 months had potential benefits on the prophylaxis against respiratory infection and diarrhea. The present data may be applicable to other infants of similar socioeconomic status.

\section{List Of Abbreviations}

WHO, World Health Organization

UNICEF, United Nations International Children's Emergency Fund

BC, bovine colostrum

RTIs, respiratory tract infections

SAE, Serious Adverse Event

$I G$, intervention group

CG, control group

$\mathrm{HC}$, head circumference

ELISA, enzyme-linked immunosorbent assays

SD, standard deviation

\section{Declarations}

Ethical Approval and Consent to participate

Eligible families in the present study were visited by a fieldworker who explained the protocol, answered questions, and obtained written informed consent from the parents. The enrollment and research plan were reviewed and approved by the institutional ethics committee of the Angel Children's Hospital Chengdu (Sichuan, China).

Consent for publication

We also have obtained the consent for publication from the infants' parents.

Availability of supporting data

The datasets used and/or analyzed during the current study are available from the corresponding author on reasonable request.

Competing interests 
The authors declare that they have no competing interests.

Funding

Not applicable.

Authors' contributions

$\mathrm{KC}$ concept and designed the study, analyzed and interpreted data, drafted and approved the final version of the manuscript. CL drafted the manuscript. $\mathrm{HC}, \mathrm{JL}, \mathrm{CZ}, \mathrm{XD}$ and $\mathrm{MZ}$ generated, collected and assembled the data.

Acknowledgements

We thank all the parents or main caregivers and their children for their participation in the study. We also thank the health care workers in field trials whose name are not mentioned above for their diligent assistance.

Authors' information

The ORCID ID of the corresponding author is: https://orcid.org/0000-0001-7455-0900.

\section{References}

[1] Liu L, Oza S, Hogan D, et al. Global, regional, and national causes of child mortality in 2000-13, with projections to inform post-2015 priorities: an updated systematic analysis. Lancet. 2015. 385(9966): 430-40.

[2] Global, regional, and national age-sex specific mortality for 264 causes of death, 1980-2016: a systematic analysis for the Global Burden of Disease Study 2016. Lancet. 2017. 390(10100): 1151-1210.

[3] Zhang J, Duan Z, Payne DC, et al. Rotavirus-specific and Overall Diarrhea Mortality in Chinese Children Younger than 5 Years: 2003 to 2012. Pediatr Infect Dis J. 2015. 34(10): e233-7.

[4] Singhal A. The global epidemic of noncommunicable disease: the role of early-life factors. Nestle Nutr Inst Workshop Ser. 2014. 78: 123-32.

[5] Newburg DS. Neonatal protection by an innate immune system of human milk consisting of oligosaccharides and glycans. J Anim Sci. 2009. 87(13 Suppl): 26-34.

[6] Morrow AL, Rangel JM. Human milk protection against infectious diarrhea: implications for prevention and clinical care. Semin Pediatr Infect Dis. 2004. 15(4): 221-8.

[7] Foster JP, Seth R, Cole MJ. Oral immunoglobulin for preventing necrotizing enterocolitis in preterm and low birth weight neonates. Cochrane Database Syst Rev. 2016. 4: CD 001816. 
[8] Korhonen H, Marnila P, Gill HS. Bovine milk antibodies for health. Br J Nutr. 2000. 84 Suppl 1: S135-46.

[9] Struff WG, Sprotte G. Bovine colostrum as a biologic in clinical medicine: a review. Part I: biotechnological standards, pharmacodynamic and pharmacokinetic characteristics and principles of treatment. Int J Clin Pharmacol Ther. 2007. 45(4): 193-202.

[10] Kelleher SL, Lönnerdal B. Immunological activities associated with milk. Adv Nutr Res. 2001. 10: 39-65.

[11] Struff WG, Sprotte G. Bovine colostrum as a biologic in clinical medicine: a review-Part II: clinical studies. Int J Clin Pharmacol Ther. 2008. 46(5): 211-25.

[12] Morris SS, Cousens SN, Kirkwood BR, Arthur P, Ross DA. Is prevalence of diarrhea a better predictor of subsequent mortality and weight gain than diarrhea incidence. Am J Epidemiol. 1996. 144(6): 582-8.

[13] Quigley JD, Martin KR, Dowlen HH. Concentrations of trypsin inhibitor and immunoglobulins in colostrum of Jersey cows. J Dairy Sci. 1995. 78(7): 1573-7.

[14] Yolken RH, Losonsky GA, Vonderfecht S, Leister F, Wee SB. Antibody to human rotavirus in cow's milk. N Engl J Med. 1985. 312(10): 605-10.

[15] Rawal P, Gupta V, Thapa BR. Role of colostrum in gastrointestinal infections. Indian J Pediatr. 2008. 75(9): 917-21.

[16] Bölke E, Jehle PM, Hausmann F, et al. Preoperative oral application of immunoglobulin-enriched colostrum milk and mediator response during abdominal surgery. Shock. 2002. 17(1): 9-12.

[17] Bagwe S, Tharappel LJ, Kaur G, Buttar HS. Bovine colostrum: an emerging nutraceutical. J Complement Integr Med. 2015. 12(3): 175-85.

[18] IGN Suwarba SS, Hendra S IS, Widiana R. The role of bovine colostrum on recovery time and length of hospital stay of acute diarrhea

in infants and children:

a double-blind randomized controlled trial. Paediatrica Indonesiana. 2006. 46(5-6): 127-133.

[19] Saad K, Abo-Elela MG, El-Baseer KA, et al. Effects of bovine colostrum on recurrent respiratory tract infections and diarrhea in children. Medicine (Baltimore). 2016. 95(37): e4560.

[20] Patel K, Rana R. Pedimune in recurrent respiratory infection and diarrhoea-the Indian experiencethe pride study. Indian J Pediatr. 2006. 73(7): 585-91.

[21] Thapa BR. Therapeutic potentials of bovine colostrums. Indian J Pediatr. 2005. 72(10): 849-52. 
[22] Howard CJ, Clarke MC, Brownlie J. Protection against respiratory infection with bovine virus diarrhoea virus by passively acquired antibody. Vet Microbiol. 1989. 19(3): 195-203.

[23] Tawfeek HI, Najim NH, Al-Mashikhi S. Efficacy of an infant formula containing anti-Escherichia coli colostral antibodies from hyperimmunized cows in preventing diarrhea in infants and children: a field trial. Int J Infect Dis. 2003. 7(2): 120-8.

[24] Ebina T, Ohta M, Kanamaru Y, Yamamoto-Osumi Y, Baba K. Passive immunizations of suckling mice and infants with bovine colostrum containing antibodies to human rotavirus. J Med Virol. 1992. 38(2): 117-23.

[25] Davidson GP, Whyte PB, Daniels E, et al. Passive immunisation of children with bovine colostrum containing antibodies to human rotavirus. Lancet. 1989. 2(8665): 709-12.

[26] Ebina T. Prophylaxis of rotavirus gastroenteritis using immunoglobulin. Arch Virol Suppl. 1996. 12: 217-23.

[27] Bogstedt A. Passive immunity against diarrhea. Acta Paediatr. 1996. 85: 125-128.

[28] Sherif ea. Bovine colostrum: Its dietary supplementation role in improvement and modulation of human immune indices. http://www.firstmilking.com/bovinecolostrumresearch.htm retrieved on 29th sept 2005 .

[29] Ylitalo S, Uhari M, Rasi S, Pudas J, Leppäluoto J. Rotaviral antibodies in the treatment of acute rotaviral gastroenteritis. Acta Paediatr. 1998. 87(3): 264-7.

[30] Caviglia GP, Ribaldone DG, Rosso C, Saracco GM, Astegiano M, Pellicano R. Fecal calprotectin: beyond intestinal organic diseases. Panminerva Med. 2018. 60(1): 29-34.

[31] Yui S, Nakatani Y, Mikami M. Calprotectin (S100A8/S100A9), an inflammatory protein complex from neutrophils with a broad apoptosis-inducing activity. Biol Pharm Bull. 2003. 26(6): 753-60.

[32] Li F, Ma J, Geng S, et al. Fecal calprotectin concentrations in healthy children aged 1-18 months. PLoS One. 2015. 10(3): e0119574.

[33] Asgarshirazi M, Shariat M, Nayeri F, Dalili H, Abdollahi A. Comparison of Fecal Calprotectin in Exclusively Breastfed and Formula or Mixed Fed Infants in the First Six Months of Life. Acta Med Iran. 2017. 55(1): 53-58.

[34] Li F, Ma J, Geng S, Wang J, Ren F, Sheng X. Comparison of the different kinds of feeding on the level of fecal calprotectin. Early Hum Dev. 2014. 90(9): 471-5. 
[35] Dorosko SM, Mackenzie T, Connor RI. Fecal calprotectin concentrations are higher in exclusively breastfed infants compared to those who are mixed-fed. Breastfeed Med. 2008. 3(2): 117-9.

[36] Bridgman SL, Konya T, Azad MB, et al. High fecal IgA is associated with reduced Clostridium difficile colonization in infants. Microbes Infect. 2016. 18(9): 543-9.

[37] Schwiertz A, Taras D, Schäfer K, et al. Microbiota and SCFA in lean and overweight healthy subjects. Obesity (Silver Spring). 2010. 18(1): 190-5.

[38] Alvaro $\mathrm{E}$, Andrieux $\mathrm{C}$, Rochet $\mathrm{V}$, et al. Composition and metabolism of the intestinal microbiota in consumers and non-consumers of yogurt. Br J Nutr. 2007. 97(1): 126-33.

[39] Prentice S. They Are What You Eat: Can Nutritional Factors during Gestation and Early Infancy Modulate the Neonatal Immune Response. Front Immunol. 2017. 8: 1641.

[40] Maywald M, Wessels I, Rink L. Zinc Signals and Immunity. Int J Mol Sci. 2017. 18(10).

\section{Figures}




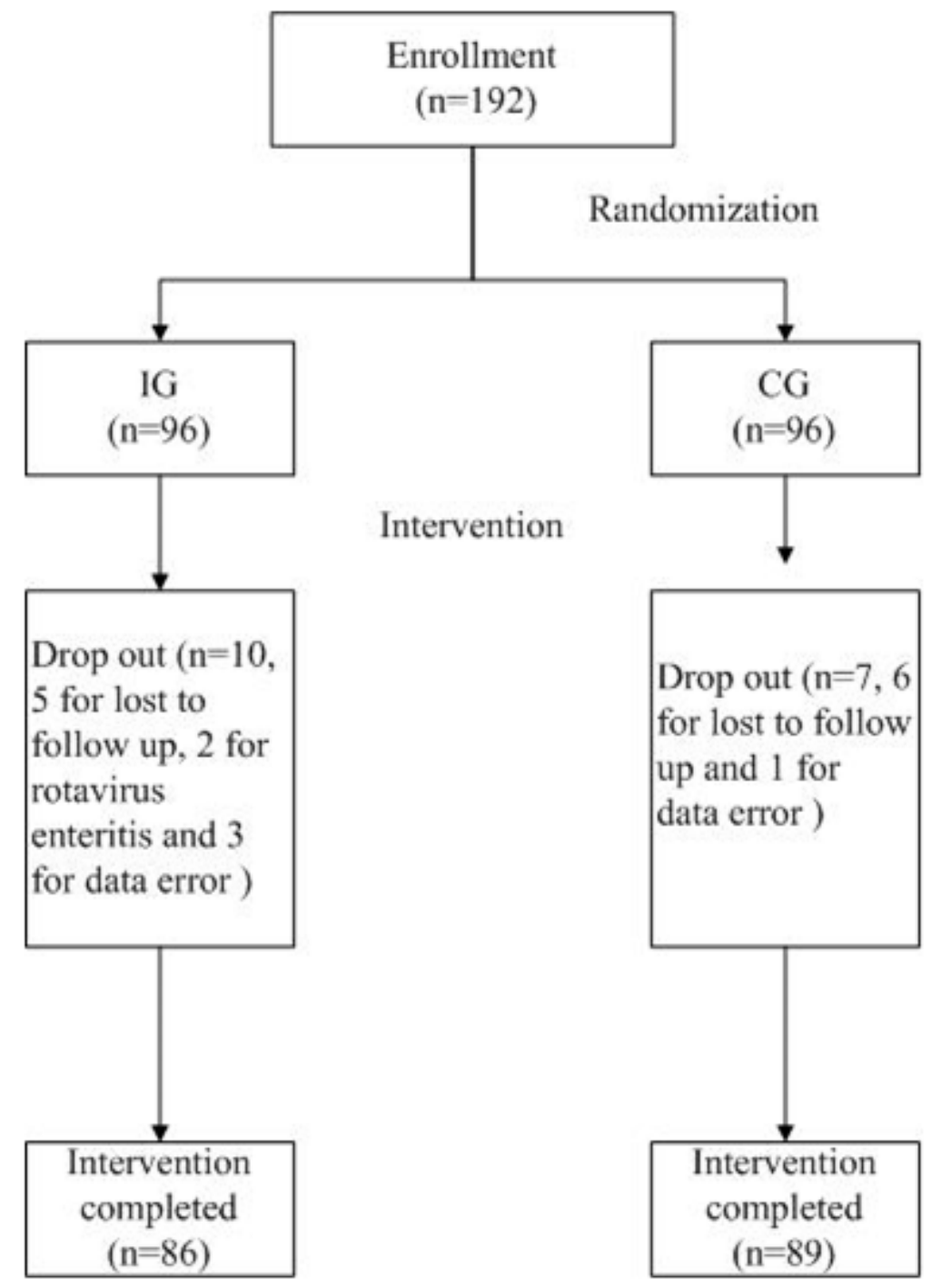

Figure 1

Flowing chart of the study.

\section{Supplementary Files}

This is a list of supplementary files associated with this preprint. Click to download.

- CONSORT2010ChecklistMSWord.doc 